\title{
The accuracy of mid upper arm circumference as an estimate of body mass index in healthy female adults
}

\author{
J. R. Houghton and S. Smith \\ Queen Margaret University, Edinburgh, EH21 6UU, UK
}

Equations developed by Powell-Tuck and Hennessy in $2003^{(1)}$ allow an estimate of body mass index (BMI) to be determined from mid upper arm circumference (MUAC). These equations are widely used in clinical practice when an individual's BMI cannot be calculated from measured height and weight. Anecdotal reports also suggest that estimated BMI derived from these equations is being used along with height to estimate the weight of some individuals who cannot be weighed, which in turn is used to calculate nutritional requirements. Secondary analysis of data for individuals who participated in the National Diet and Nutrition Survey (aged 65 years or over) suggests that there is a substantial variability in BMI predicted from an individual MUAC ${ }^{(2)}$. However there appears to be a lack of such data in younger adults. Therefore a pilot study aimed at assessing the accuracy of BMI estimated using the Powell-Tuck and Hennessy equations and the subsequent accuracy of estimated weight, within a younger healthy female adult population was undertaken.

Subjects who volunteered had their age recorded, and actual height, weight and MUAC measured. From this data actual BMI, estimated BMI and estimated weight were calculated. The relationship between actual BMI and MUAC, actual BMI and estimated BMI were explored using pearsons correlation. The accuracy of estimated BMI with actual BMI and estimated weight with actual weight were determined using Bland-Altman limits of agreement.

29 subjects volunteered with a mean age of 26.1 years (standard deviation (SD) 10.2 years) and a mean actual BMI of $23.1 \mathrm{Kg} / \mathrm{m}^{2}(\mathrm{SD}$ $\left.4.3 \mathrm{Kg} / \mathrm{m}^{2}\right)$. A strong positive $(\mathrm{r}=0.912)$, statistically significant $(\mathrm{p}<0.001)$ correlation between actual BMI and MUAC was identified, as was a strong positive correlation between actual BMI and estimated BMI $(r=0.896)$. Bland-Altman analysis revealed a mean difference of $-0.08 \mathrm{Kg} / \mathrm{m}^{2}$ for estimated BMI versus actual BMI and a mean difference of $-0.16 \mathrm{Kg}$ for estimated weight versus actual weight. However the $95 \%$ confidence intervals for estimated BMI versus actual BMI were $-5.64 \mathrm{Kg} / \mathrm{m}^{2}$ to $4.10 \mathrm{Kg} / \mathrm{m}^{2}$ and $-13.23 \mathrm{Kg}$ to $12.91 \mathrm{Kg}$ for estimated weight versus actual weight.

The results demonstrate a strong positive relationship between BMI and MUAC, and between actual BMI and estimated BMI. However they also demonstrated that at an individual level both estimated BMI and estimated weight could be significantly over or under the actual value, to a level that could affect clinical practice by altering BMI classification and/or calculated nutritional requirements.

Although further work is required to determine the accuracy of Powell-Tuck and Hennessy equations in other populations and to potentially derive more accurate equations, practitioners should be aware of the potential inaccuracies of using the Powell-Tuck and Hennessy equations to estimate an individual's BMI or to derive an individual estimation of weight.

1. Powell-Tuck J \& Hennessy EM (2003) A Comparison of mid upper arm circumference, body mass index and weight loss as indices of undernutrition in acutely hospitalised patients. Clil Nutr 22, 307-312.

2. Elia M (Editor) (2003) The 'MUST' Report. Nutritional Screening of adults: a multidisciplinary responsibility. BAPEN UK. 